AIAA-2001-2531

\title{
NAVIER-STOKES OPTIMIZATION OF SUPERSONIC WINGS WITH FOUR DESIGN OBJECTIVES USING EVOLUTIONARY ALGORITHM
}

\author{
Daisuke SASAKI ${ }^{*}$, Shigeru OBAYASHI ${ }^{\dagger}$ and Kazuhiro NAKAHASHI ${ }^{\ddagger}$ \\ Tohoku University, Sendai 980-8577, JAPAN
}

\begin{abstract}
The design optimization of wings for supersonic transport by means of Multiobjective Evolutionary Algorithms is presented. Three objective functions are first considered to minimize the drag for transonic cruise, the drag for supersonic cruise and the bending moment at the wing root at the supersonic condition. The wing shape is defined by planform, thickness distributions and warp shapes in total of 66 design variables. A Navier-Stokes code is used to evaluate the aerodynamic performance at both cruise conditions. Based on the results, the optimization problem is further revised. The definition of the thickness distributions is given more precisely by adding control points. In total 72 design variables are used. The fourth objective function to minimize the pitching moment is added. The results of the revised optimization are compared with the three-objective optimization results as well as NAL's design. Two Pareto solutions are found superior to NAL's design for all four objective functions. The planform shapes of those solutions are "Arrow wing" type.
\end{abstract}

\section{Introduction}

To develop a new Supersonic Transport (SST), many researches have been performed. Especially in Japan, National Aerospace Laboratory (NAL) is conducting the scaled supersonic experimental airplane project. ${ }^{1}$

For a new SST design, there exist many technical difficulties to be overcome. L/D must be improved, and the sonic boom should be prevented. However, there is a severe tradeoff between reducing the drag and boom. As a result, a new SST is expected to cruise at a supersonic speed only over the sea and to cruise at a transonic speed over the land. This means the important design objectives are not only to improve a supersonic cruise performance but also to improve a transonic one. For example, a large sweep

\footnotetext{
* Graduate student, Institute of Fluid Science

${ }^{\dagger}$ Associate professor, Institute of Fluid Science, Associate Fellow AIAA

${ }^{\ddagger}$ Professor, Department of Aeronautics and Space

Engineering, Associate Fellow AIAA
}

Copyright () 2001 by the American Institute of Aeronautics and Astronautics, Inc. All rights reserved. angle can reduce the wave drag, but it limits the low-speed aerodynamic performance. Therefore, there are many tradeoffs to be addressed in designing a SST.

To identify such tradeoffs efficiency, the multiobjective (MO) optimization must be performed. MO optimization seeks to optimize the components of a vector-valued objective function unlike the single objective optimization. Pareto solutions, which are members of the Pareto-optimal set obtained by solving MO problems, represent tradeoffs among multiple objectives. Since an application of Evolutionary Algorithms (EAs) to MO problem has many advantages, such methods have been increasingly used in aerodynamic optimization problems. $^{2-4}$ Multiobjective Genetic Algorithms (MOGAs) can sample multiple Pareto solutions efficiency and effectively. Since GAs seek optimal solutions in parallel using a population of design candidates, MOGAs can identify multiple Pareto solutions at the same time without specifying weights between objectives. Objective functions can be evaluated by the existing CFD solver without any modification.

This paper presents the multipoint aerodynamic optimization of a wing shape for SST at both supersonic and transonic cruise conditions by MOGAs. Both aerodynamic drags will be minimized under lift constraints, and the bending moment at the wing root will also be minimized to prevent all the Pareto solutions having impractically large aspect ratios. A Navier-Stokes solver is used to evaluate the wing performance at both conditions. In the present design optimization, planform shapes, camber, thickness distributions and twist distributions are parameterized. The resulting Pareto solutions are analyzed and compared with NAL's design and the previous inviscid optimization results. ${ }^{3}$ As a result, a few problems are revealed in the problem definition.

The revised multiobjective optimization is then performed to obtain more realistic wing designs. In the revised optimization, the minimization of a pitching moment is added as the fourth objective function to reduce the pitching moment associated with a highly swept wing. The definition of the thickness distributions is also improved, because the previous definition, which appears quite suitable for inviscid optimization, has a problem with viscous flows. To improve the solutions quality further, Adaptive Range Multiobjective Genetic Algorithms 
(ARMOGAs) are applied. Final Pareto solutions will be examined in detail.

In this study, a Navier-Stokes solver is used to evaluate the objective functions for optimization process. To evolve a population of design candidates, the enormous calculation time is required. However, all the solutions show tradeoffs in contrast to the single optimization where the solution is merely a point in the design space. Therefore, detailed tradeoff study can be performed. To alleviate the required computational time, parallel computing is performed.

\section{Evolutionary Multiobjective Optimization}

EAs, in particular GAs, are based on the theory of evolution, where a biological population evolves over generations to adapt to an environment by selection, crossover and mutation. In design optimization problems, fitness, individual and genes correspond to an objective function, design candidate and design variables, respectively.

\subsection{Multiobjective GAs (MOGAs)}

GAs search from multiple points in the design space simultaneously and stochastically, instead of moving from a single point deterministically like gradient-based methods. This feature prevents design candidates from settling in local optimum. Moreover, GAs do not require computing gradients of the objective function. These characteristics lead to following three advantages of GAs: 1, GAs have capability of finding global optimal solutions. 2, GAs can be processed in parallel. 3, High fidelity CFD codes can easily be adapted to GAs without any modification.

GAs have been extended to solve MO problems successfully. ${ }^{5}$ GAs use a population to seek optimal solutions in parallel. This feature can be extended to seek Pareto solutions in parallel without specifying weights between the objective functions. The resultant Pareto solutions represent global tradeoffs. Therefore, MOGAs are quite unique and attractive methods to solve MO problems.

Figure 1 shows the flowchart of MOGAs used in the present study. The following describes genetic operators employed here in brief. Traditionally, GAs use binary representation of design variables. For real function optimizations like the present aerodynamic optimization, however, it is more straightforward to use real numbers. Thus, the floating-point representation is used here. Selection is based on the Pareto ranking method and fitness sharing. ${ }^{5}$ Each individual is assigned to its rank according to the number of individuals that dominate it. A standard fitness sharing function is used to maintain the diversity of the population. To find the Pareto solutions more effectively, the so-called best- $N$ selection $^{6}$ is also coupled with. Blended crossover $(B L X-\alpha)^{7}$ described below is adopted. This operator generates children on a segment defined by two parents and a user specified parameter $\alpha$. The disturbance is added to new design variables at a mutation rate of $20 \%$. If the mutation occurs, new design variables are obtained as

Child $1=\gamma \cdot$ Parent $1+(1-\gamma) \cdot$ Parent $2+m \cdot($ ran2- 0.5$)$

Child $2=(1-\gamma) \cdot$ Parent $1+\gamma \cdot$ Parent $2+m \cdot($ ran3- 0.5$)$

$\gamma=(1+2 \alpha) \cdot \operatorname{ran} 1-\alpha$

where Child1,2 and Parent 1,2 denote encoded design variables of the children (members of the new population) and parents (a mated pair of the old generation), respectively. The random numbers ran $1-3$ are uniform random number in $[0,1]$ and $m$ is set to $10 \%$ of the given range of each design variable.

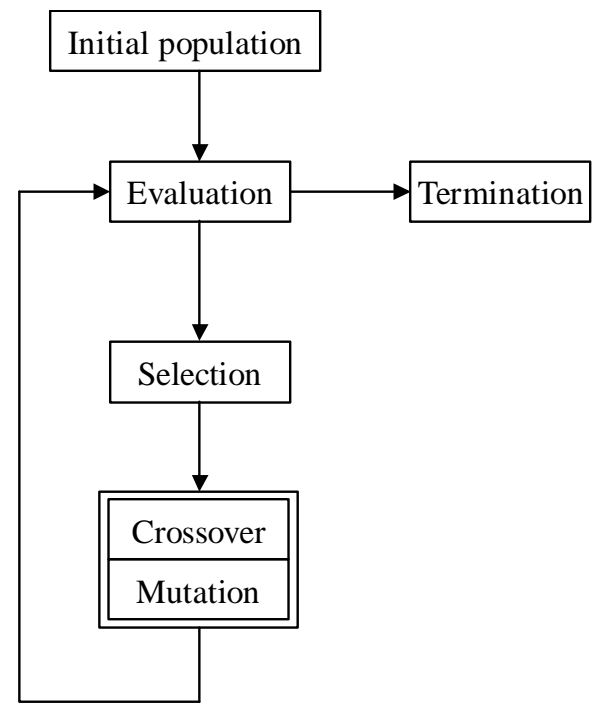

Fig. 1. Flowchart of MOGAs

\subsection{Adaptive Range MOGAs (ARMOGAs)}

To reduce the large computational burden for CFD evaluation, the reduction of the total number of evaluations is desired. On the other hand, a large search space is essential for real parameter problems. Adaptive Range GAs (ARGAs) originally proposed by Arakawa and Hagiwara are quite unique and effective for solving such problems. $^{8}$ Oyama developed real-coded ARGAs and applied them to the transonic wing optimization. ${ }^{9}$ In this study, ARGAs are extended to solve MO problems.

The main difference between ARGAs and conventional GAs is the introduction of the range adaptation based on the population statistics. The flowchart of ARGAs is shown in Fig. 2. Population candidates are reinitialized every $M$ generations for the range adaptation so that the population focuses on promising regions. Encoding method is also revised by using normal distribution. The real value of $i$-th design variable $p_{i}$ is encoded to a real number $r_{i}$ defined in $(0,1)$ so that $r_{i}$ is equal to the integrations of the normal distribution from $-\infty$ to $p n_{i}$, 
$r_{i}=\int_{-\infty}^{p n_{i}} N(0,1)(z) d z$

$p n_{i}=\left(p_{i}-\mu_{i}\right) / \sigma_{i}$

where the average $\mu_{i}$ and the standard deviation $\sigma_{i}$ of $i$-th design variable are calculated by sampling the top half of the previous population to promote the population toward search regions of high fitness. Due to the range adaptation, ARMOGAs are expected to search optimal solutions efficiently in the large design space. A schematic view of this encoding is illustrated in Fig. 3.

In this study, ARGAs have to deal with multiple Pareto solutions for the MO optimization. The basis of ARMOGAs is the same as ARGAs, but a straightforward extension may damage the diversity of the population. To maintain the diversity of solution candidates, the normal distribution for encoding have to be revised by the introduction of a plateau as shown in Fig. 4. The search region is partitioned into three parts (i, ii, iii) and controlled by two parameters $\alpha$ and $\beta$ where $\alpha$ determines the ratio of population in region (i) and $\beta$ represents the width of the plateau. In the regions (i) and (iii), ARGAs' encoding method is used. In contrast, the region (ii) adopts the conventional real-number encoding method.

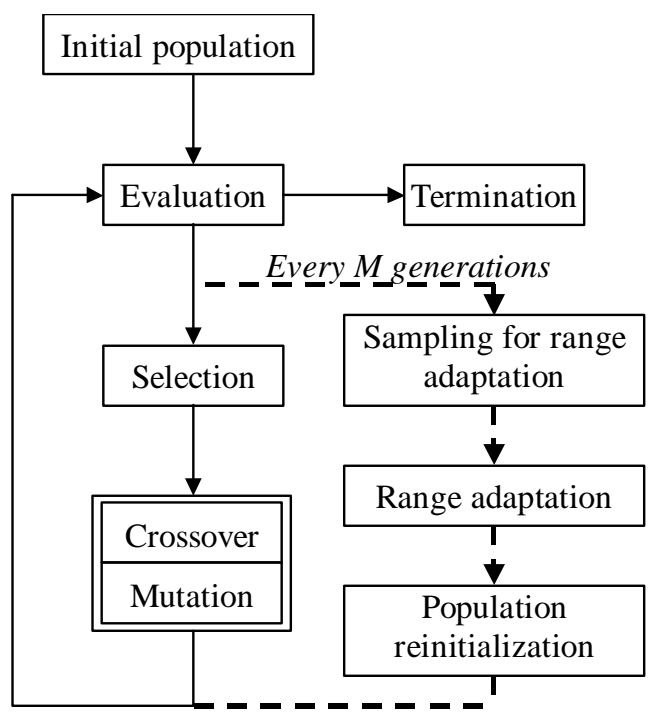

Fig. 2. Flowchart of ARMOGAs

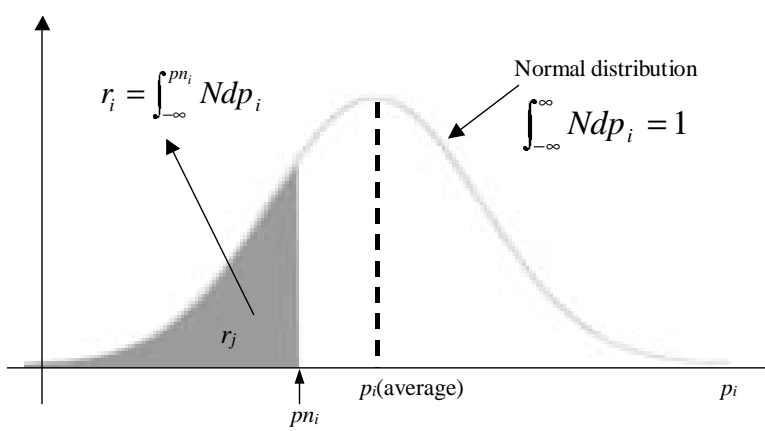

Fig. 3. Encoding based on normal distribution for ARGAs

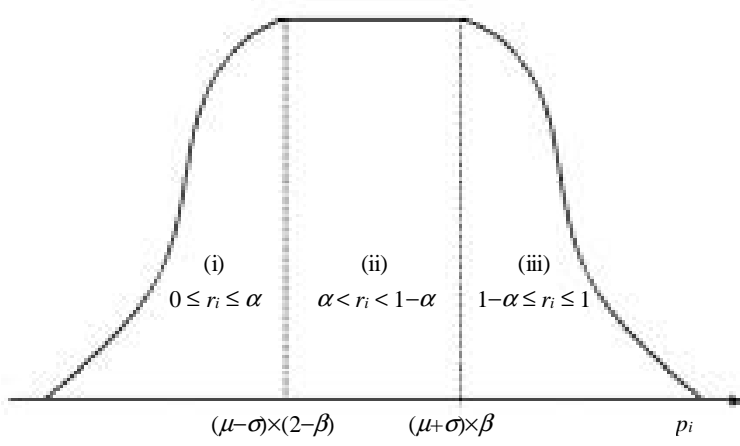

Fig. 4. Encoding with plateau for ARMOGAs

\subsection{CFD Evaluation}

To account for the viscous effect, the three-dimensional, compressible, thin-layer Navier-Stokes code is used to evaluate aerodynamic performances of three-dimensional wings at both transonic and supersonic conditions. This Navier-Stokes code employs total-variationdiminishing type upwind differencing and the lower-upper factored symmetric Gauss-Seidel scheme. ${ }^{10}$ An algebraic mixing length turbulence model by Baldwin and Lomax is adopted. ${ }^{11}$ To accelerate the convergence, the multigrid method is also used. ${ }^{12}$

Taking advantage of the characteristic of GAs, the present optimization is parallelized on SGI ORIGIN2000 at the Institute of Fluid Science, Tohoku University. The system has 640 PE's with peak performance of 384 GFLOPS and 640 GB of memory. The master PE manages the optimization process, while the slave PE's compute the Navier-Stokes code. The population size used in this study was set to 64 so that the process was parallelized with 32-128 PE's depending on the availability. It should be noted that the parallelization was almost $100 \%$ because almost all the CPU time was dominated by Navier-Stokes computations. The present optimization requires about six hours per each generation when parallelized on 128 PE's.

\section{Multiobjective Aerodynamic Optimization}

\subsection{Formulation of Three-Objective Optimization}

The objective functions used here can be stated as follows:

1. Drag coefficient at transonic cruise, $C_{D, t}$

2. Drag coefficient at supersonic cruise, $\mathrm{C}_{\mathrm{D}, \mathrm{s}}$

3. Bending moment at the wing root at supersonic cruise condition, $\mathrm{M}_{\mathrm{B}}$

In the present optimization, all three objective functions are to be minimized. The bending moment represents the lateral moment that acts at the wing 
root as illustrated in Fig. 5 and it is calculated by directly integrating the computed pressure load at the supersonic condition. The present optimization is performed at two design points for the transonic and supersonic cruises. Each flow condition and the target lift coefficient are described as

1. Transonic cruising Mach number, $M_{\infty, t}=0.9$

2. Supersonic cruising Mach number, $M_{\infty, \mathrm{s}}=2.0$

3. Target lift coefficient at transonic cruising condition, $\mathrm{C}_{\mathrm{L}, \mathrm{t}}=0.15$

4. Target lift coefficient at supersonic cruising condition, $\mathrm{C}_{\mathrm{L}, \mathrm{s}}=0.10$

5. Reynolds number based on the root chord length at both conditions, $\operatorname{Re}=1.0 \times 10^{7}$

Flight altitude is assumed at $10 \mathrm{~km}$ for the transonic cruise and at $15 \mathrm{~km}$ for the supersonic cruise. To maintain lift constraints, the angle of attack is computed for each configuration by using $\mathrm{C}_{\mathrm{L}_{\alpha}}$ obtained from the finite difference. Thus, three Navier-Stokes computations per evaluation are required. During the aerodynamic optimization, wing area is frozen at a constant value.

Design variables are categorized to planform, airfoil shapes and the wing twist. The wing planform is determined by six design variables as shown in Fig. 5 and constraints for the planform are summarized in Table 1. A chord length at the wing tip is determined accordingly because of the fixed wing area. Airfoil shapes are composed of its thickness distribution and camber line. The thickness distribution is represented by a Bézier curve defined by nine polygons as shown in Fig. $6{ }^{13}$ The wing thickness is constrained for structural strength as described in Table 1. The thickness distributions are defined at the wing root, kink and tip, and then linearly interpolated in the spanwise direction. The total number of polygons is 27 for the entire thickness distribution.

The camber surfaces composed of the airfoil camber lines are defined at the inboard and outboard of the wing separately. Each surface is represented by the Bézier surface defined by four polygons in the chordwise direction and three in the spanwise direction. For instance, Figure 7 shows the camber line with its control points at the root. It is concave only at the root and it becomes convex at the other spanwise locations similar to the warp design based on the linearized theory. The number of polygons that defines two camber surfaces is 20 in total. Finally, the wing twist is represented by a B-spline curve with six polygons as shown in Fig. 8. As a result, 66 design variables are used to define a whole wing shape. In Fig. 9, a three-dimensional wing with computational structured grid is illustrated.

MOGAs (Section 2-1) are used to seek the Pareto solutons of this three-objective optimization. In this study, the population is set to 64 and the parameter $\alpha$, which is used in the selection process, is set to 0.5 except for the planform definition design variables.
In the case of the six planform design variables, $\alpha$ is set to 0.0 to prevent possible divergence of computation for the new configuration.

Table 1. Summary of constraints

(a) Constraints for planform shape

\begin{tabular}{l|c}
\hline Chord length at root & $10<C_{\text {root }}<20$ \\
\hline Chord length at kink & $3<C_{\text {kink }}<15$ \\
\hline Inboard span length & $2<b_{\text {in }}<7$ \\
\hline Outboard span length & $2<b_{\text {out }}<7$ \\
\hline Inboard sweep angle $(\mathrm{deg})$ & $35<\alpha_{\text {root }}<70$ \\
\hline Outboard sweep angle $(\mathrm{deg})$ & $35<\alpha_{\text {kink }}<70$ \\
\hline Wing area & $\mathrm{S}=60$ \\
\hline Chord length at tip & $1<C_{\text {tip }}<10$ \\
\hline Chord length & $C_{\text {tip }}<C_{\text {kink }}<C_{\text {root }}$ \\
\hline Span length & $b_{\text {out }}<b_{\text {in }}$ \\
\hline Sweep angle & $\alpha_{\text {kink }}<\alpha_{\text {root }}$ \\
\hline
\end{tabular}

(b) Constraints for thickness distribution

\begin{tabular}{l|c}
\hline Maximum thickness $(\%)$ & $3<\mathrm{ZP}_{4}<4$ \\
\hline Maximum thickness location $(\%)$ & $15<\mathrm{XP}_{4}<70$ \\
\hline Continuous first derivative at $\mathrm{P}_{4}$ & $\mathrm{Z}_{\mathrm{P}_{3}}=\mathrm{Z}_{4}=\mathrm{Z}_{5}$ \\
\hline $\begin{array}{l}\text { Continuous first derivative } \\
\text { at leading edge }\end{array}$ & $\mathrm{XP}_{0}=\mathrm{XP}_{1}$ \\
\hline
\end{tabular}

(c) Constraints for camber distribution

\begin{tabular}{l:c}
\hline Negative camber at wing root & $\mathrm{Z}_{\mathrm{C} 01}, \mathrm{Z}_{\mathrm{C} 02}<0$ \\
\hline \multirow{2}{*}{ Positive camber } & $\mathrm{Z}_{\mathrm{C}_{21},}, \mathrm{Z}_{\mathrm{C}_{22},}, \mathrm{Z}_{\mathrm{C}_{31}}$, \\
& $\mathrm{Z}_{\mathrm{C} 32}, \mathrm{Z}_{\mathrm{C} 41}, \mathrm{Z}_{\mathrm{C} 42}<0$ \\
\hline
\end{tabular}

(d) Constraints for twist distribution

\begin{tabular}{l:c}
\hline Twist angle at tip & $3.5<\mathrm{Z}_{\mathrm{T} 1}-\mathrm{Z}_{\mathrm{T} 4}<7.5$ \\
\hline $\begin{array}{l}\text { Monotone decrease of twist } \\
\text { angle }\end{array}$ & $\mathrm{Z}_{\mathrm{T} 2}>\mathrm{Z}_{\mathrm{T} 3}$ \\
\hline Control point location $(\mathrm{y})$ & $\mathrm{Y}_{\mathrm{T} 0}=-0.1, \mathrm{Y}_{\mathrm{T} 5}=1.1$ \\
\hline
\end{tabular}

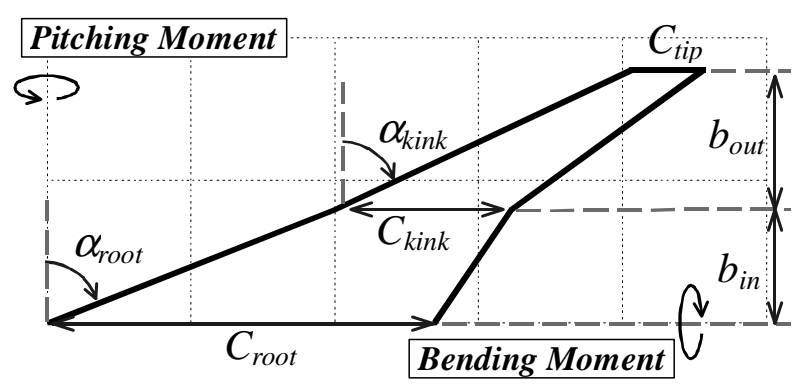

Fig. 5. Wing planform definition and schematic view of moment axes 


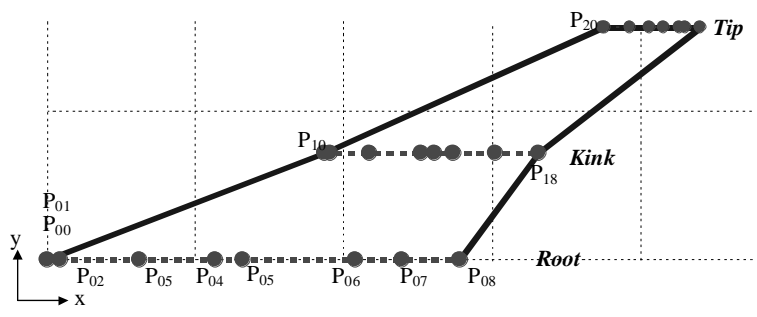

(a) Control point distribution in the wing planform

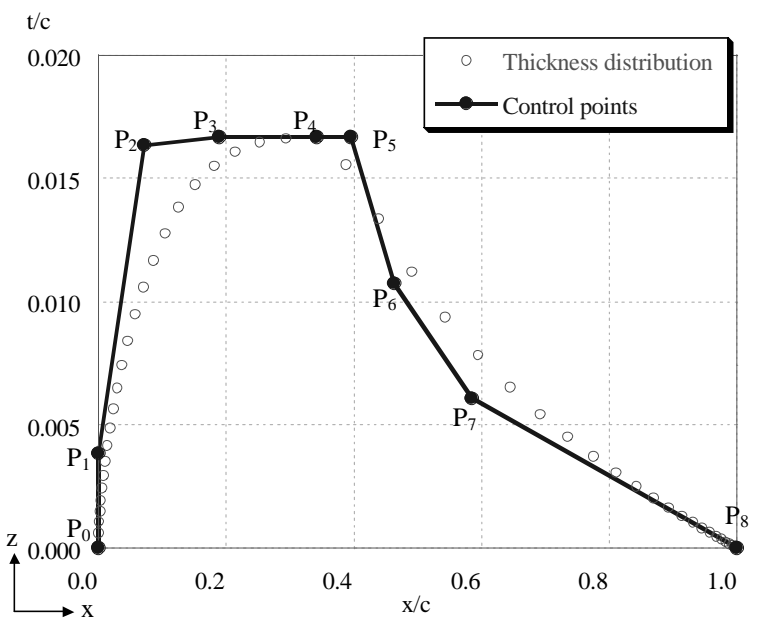

(b) Chordwise control point distribution

Fig. 6. Wing thickness definition

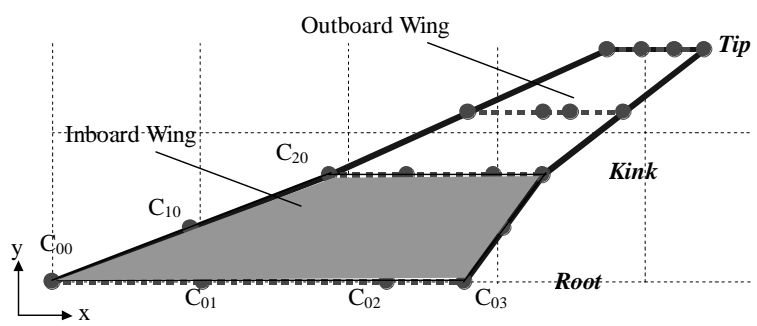

(a) Control point distribution in the wing planform

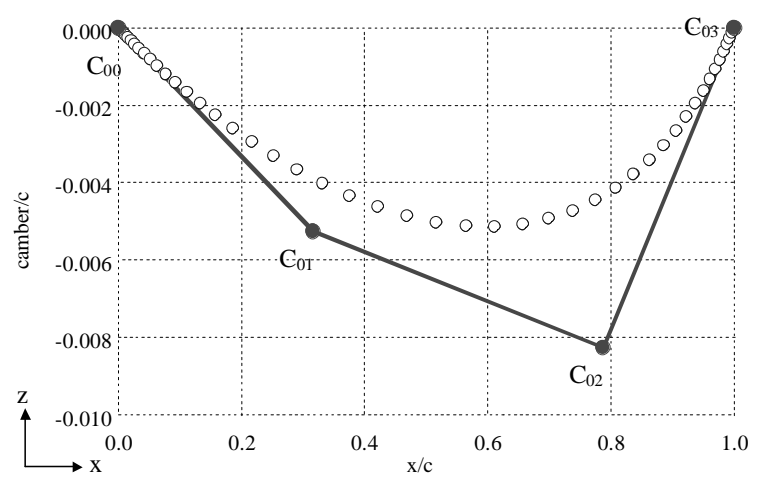

(b) Chordwise control point distribution

Fig. 7. Wing camber surface definition

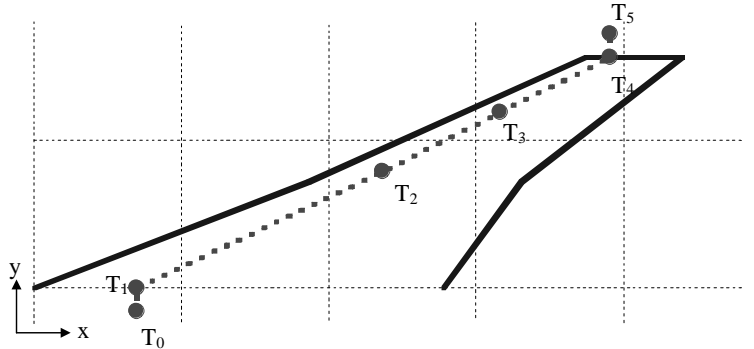

(a) Control point distribution in the wing planform

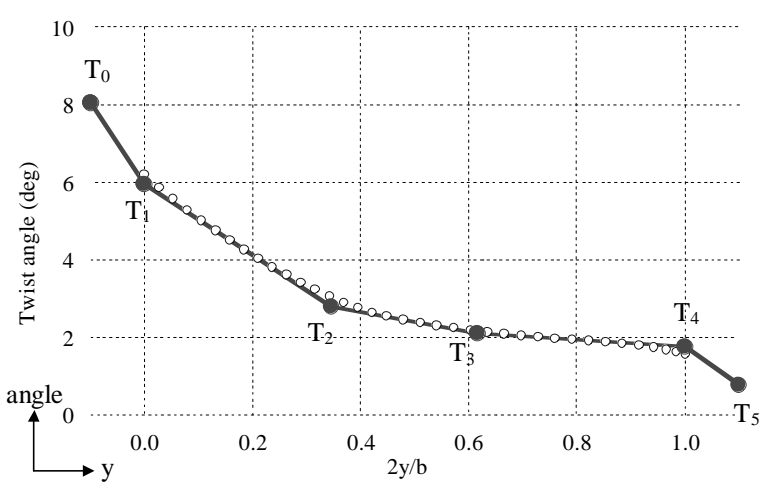

(b) Spanwise control point distribution

Fig. 8. Wing twist definition

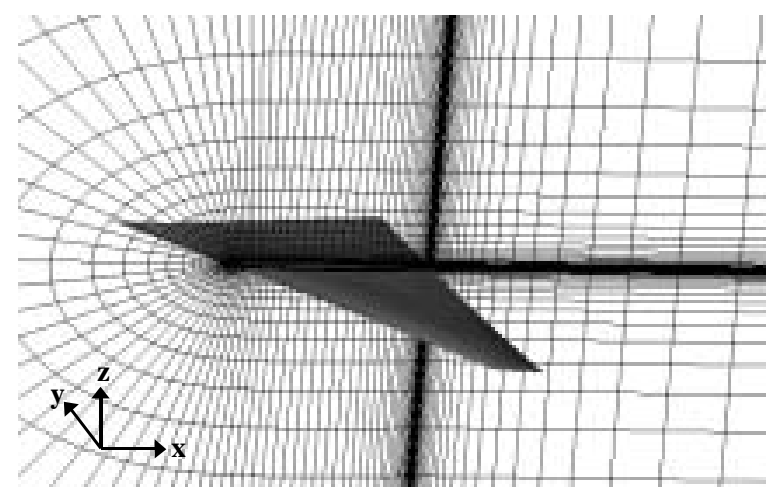

Fig. 9. Wing with structured grid in C-H topology

\subsection{Overview of Resulting Pareto Solutions}

The evolution was computed for 30 generations. After that, all the solutions evaluated were sorted again to find the final Pareto front. As a result, the final Pareto solutions were obtained in the three-dimensional objective function space as shown in Fig. 10. The tradeoff surface with the objective functions is exhibited in the figure. Four typical planform shapes are also plotted for solutions at $\mathrm{C}_{\mathrm{D}, \mathrm{t}}$ minimum, $\mathrm{C}_{\mathrm{D}, \mathrm{s}}$ minimum, bending moment minimum and near the center of the front. The extreme Pareto solutions, three planform shapes that minimize the respective objective functions, appear physically reasonable.

To present tradeoffs between the objectives more clearly, Pareto solutions are projected into the two-dimensional plane as shown in Figs. 11-13. Figures 11 and 12 present the tradeoffs between 
transonic and supersonic drag coefficients. The solutions are labeled by the aspect ratio and the taper ratio in Figs. 11 and 12, respectively. In Fig. 11, wings with larger aspect ratios achieve lower drag coefficients as expected. Figure 12 shows that the wings with the taper ratios smaller than 0.4 have good aerodynamic performances, but further decrease of the taper ratio does not correspond to the reduction of cruising drag directly. On the other hand, the wings with the taper ratios larger than 0.4 have the lower bending moments and poor aerodynamic performances as shown in Fig. 13.

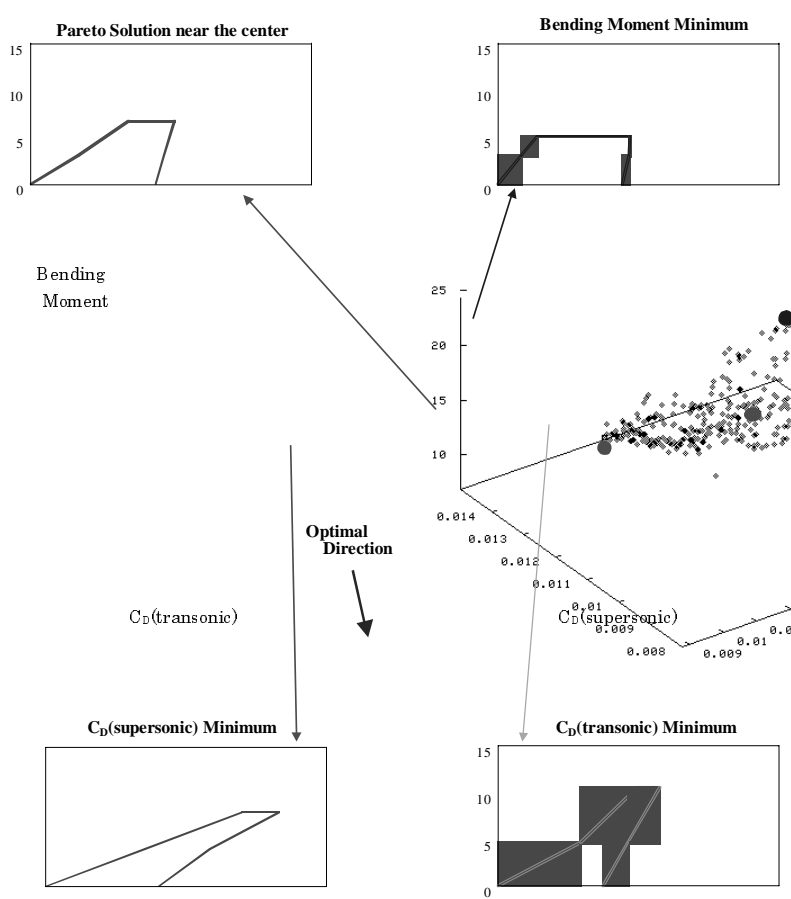

Fig. 10. Pareto front in the objective function space and typical planform shapes

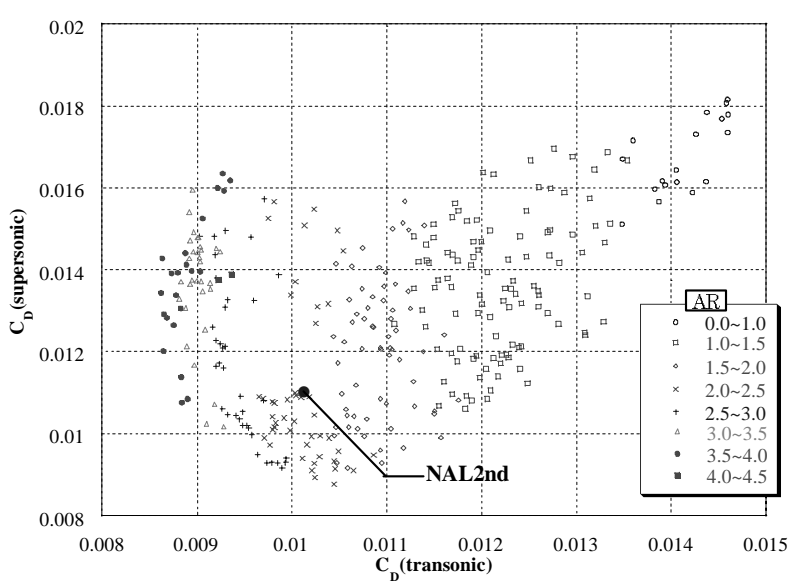

Fig. 11. Projection of Pareto front to supersonic and transonic drag tradeoffs labeled according to aspect ratios (NAL's design is plotted here for a comparison although it is not Pareto optimal.)

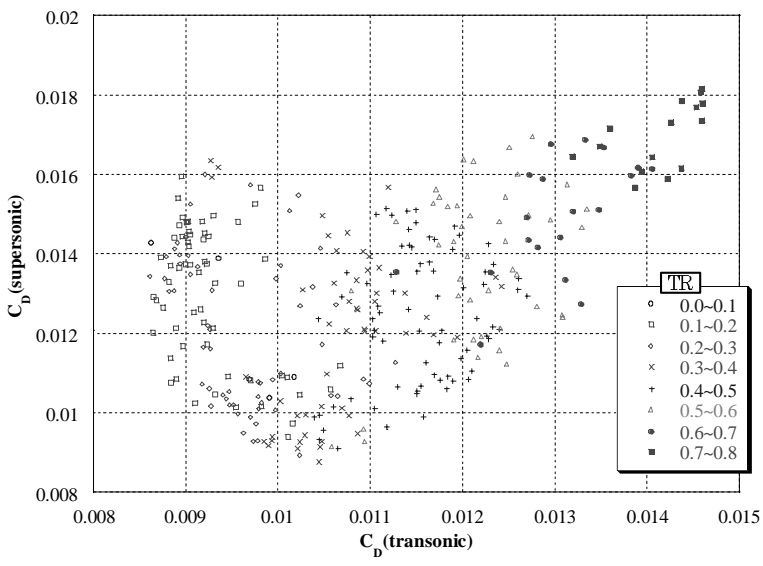

Fig. 12. Projection of Pareto front to supersonic and transonic drag tradeoffs labeled according to taper ratios

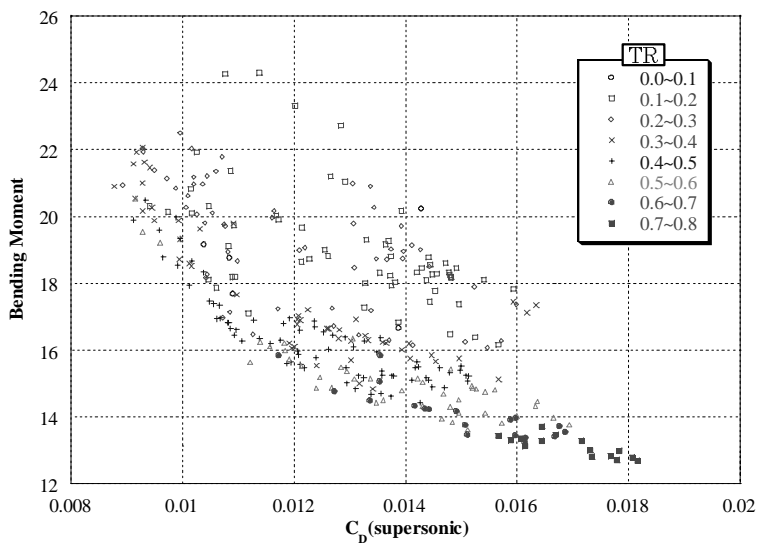

Fig. 13. Projection of Pareto front to bending moment and supersonic drag tradeoffs labeled according to taper ratios

\subsection{Comparison with NAL's Second Design}

NAL SST Design Team already finished the fourth aerodynamic design for the experimental supersonic airplane to be launched in 2002. To summarize their design concepts briefly, the first design determined the planform shapes among 99 candidates, and the second design was performed by the warp optimization based on the linearized theory. The third design aimed a natural-laminar-flow (NLF) wing by an inverse method using a Navier-Stokes code. Finally, the fourth design was performed for a wing-fuselage configuration. ${ }^{14}$ Because a fully developed turbulence is assumed in the present Navier-Stokes computations, it is improper to compare the present Pareto solutions to NAL's NLF wing design. Therefore, NAL's second design is chosen for a comparison.

Table 2 summarizes comparisons of a Pareto solution (3V) with NAL's second design. The aerodynamic calculation of NAL's second design is performed here by using the same Navier-Stokes solver. The Pareto solution presented here are superior to NAL's second design in all three objectives. Figure 14 shows the wing planforms of 
the two, indicating a large difference of planform shapes between the present solutions and NAL's design. (The inviscid result will be discussed in the next section.) The present planforms are similar to the "arrow wing" planform and the NAL's planform is similar to the conventional "delta wing" planform.

The thickness distributions of the two wings are shown in Fig. 15 as well as the previously obtained inviscid solution. The thickness distribution of NAL's design is simply taken from an existing NLF airfoil. In contrast, the present optimization is performed under a fully turbulent flow with the thickness constraint. Therefore, the maximum thickness appears near the leading edge. Then, the thickness is reduced toward the trailing edge to prevent the rapid growth of the boundary layer.

Table2. Aerodynamic performances between the selected Pareto solution (3V) and NAL's second design

\begin{tabular}{c||c:c:c}
\hline & $\mathrm{C}_{\mathrm{D}, \mathrm{t}}$ & $\mathrm{C}_{\mathrm{D}, \mathrm{s}}$ & $\mathrm{M}_{\mathrm{B}}$ \\
\hline \hline 3V & 0.01004036 & 0.01093742 & 18.21 \\
\hline NAL2nd & 0.01010175 & 0.01097646 & 18.23 \\
\hline
\end{tabular}

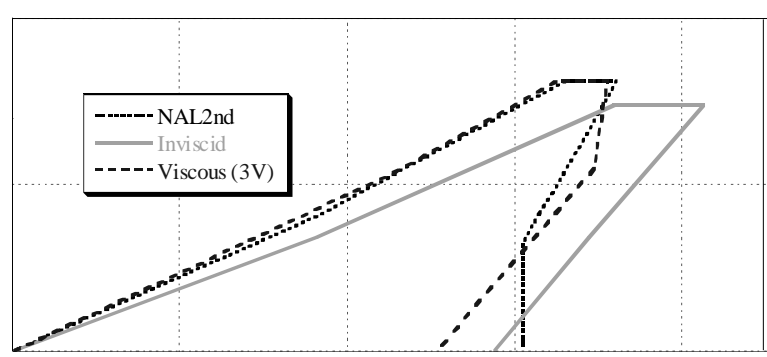

Fig. 14. Comparison of planform shapes of the viscous $(3 \mathrm{~V})$ and inviscid designs with NAL's design

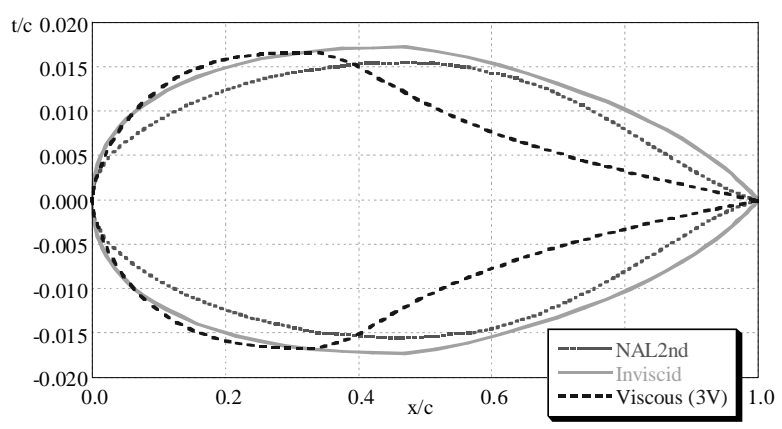

Fig. 15. Comparison of thickness distributions at the wing root of the viscous $(3 \mathrm{~V})$ and inviscid designs with NAL's design

\subsection{Difference between viscous and inviscid calculations}

The present viscous designs $(3 \mathrm{~V})$ are compared with the inviscid designs computed previously. ${ }^{3} \mathrm{By}$ comparing the two optimization results, the difference of the wing shapes due to the viscous effect becomes clear. The Pareto solutions that are found to outperform NAL's design in all three objectives at both cases are selected for the comparison.

A comparison of the planform shapes is shown in Fig. 14. Both planform shapes are similar to the "arrow wing" planform, but the shapes are slightly different. The viscous design has a less sweep angle and a less taper ratio than the inviscid design. A highly swept wing tends to have a flow separation near the wing tip. The present viscous design appears better than the inviscid design to prevent the possible tip separation.

Figure 15 shows a comparison of the thickness distributions at the root. It shows the quite different distributions. In the viscous case, the wing is thicker near the leading edge and thinner near the trailing edge. However, in the inviscid case, the wing is very thick. The $\mathrm{C}_{\mathrm{p}}$ distributions shown in Fig. 16 explain their difference. In the inviscid case, the $C_{p}$ distribution has a discontinuity at the trailing edge, and therefore it generates the lift even at the trailing edge. However, such a thick airfoil probably causes a flow separation. It is important to consider the viscous effect for designing thickness distributions.

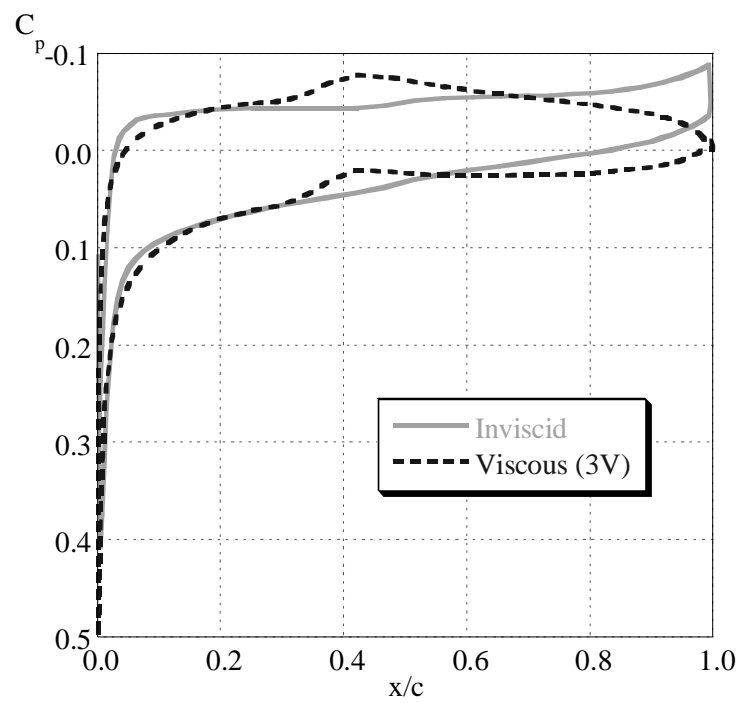

Fig. 16. Comparison of $\mathrm{C}_{\mathrm{p}}$ distributions at the wing root of the viscous $(3 \mathrm{~V})$ and inviscid designs

\section{Revised Multiobjective Aerodynamic Optimization}

\subsection{Formulation of Four-Objective Optimization}

The three-objective optimization described in the previous section obtained good tradeoff surfaces and the Pareto solution that outperformed the NAL's design in all three objectives. However the results also showed some problems. First, although "arrow wing" type planform is known to be good for supersonic aerodynamics, it is also known to have aeroelastic and control problems due to a large sweep 
angle. A pitching moment of the wing should be monitored. Second, the design results contain discontinuities in the second derivative of the wing thickness distribution at the maximum thickness location as shown in Fig. 15. This leads to concerns of the designer for the possible boundary layer separation at off-design conditions.

Therefore, the optimization problem is redefined in this section. To reduce the pitching moment and investigate its effect, the minimization of the pitching moment is added as the fourth objective function. The pitching moment is measured at the leading edge of the root. The overview of pitching axis is also illustrated in Fig. 5. In addition, since the previous thickness definition only enforces the continuity in the first derivative at the maximum thickness location (Table 1 and Fig. 6), not only the first derivative but also the second derivative is constrained here. Two polygons, which have the same distance from the maximum thickness position, are added to the original definition of the thickness as shown in Fig. 17. The constraints for the revised thickness definition are summarized in Table. 3. The total number of design variables becomes 72 .

Except for these two improvements, all the design conditions are the same as the first optimization case. The four objective functions to be minimized are

1. Drag coefficient at transonic cruise, $C_{D, t}$

2. Drag coefficient at supersonic cruise, $C_{D, s}$

3. Bending moment at the wing root at supersonic cruise condition, $\mathrm{M}_{\mathrm{B}}$

4. Pitching moment at supersonic cruise condition, $\mathrm{M}_{\mathrm{P}}$

The present four-objective optimization is performed by ARMOGAs (Section 2-2). The standard genetic operators are the same as the previous. A population is also set to 64 . The range adaptation is performed every 10 generations starting from the $15^{\text {th }}$ generation. Two parameters $\alpha$ and $\beta$, which control the encoding method of ARMOGAs, are set to 0.4 and 1.0 , respectively.

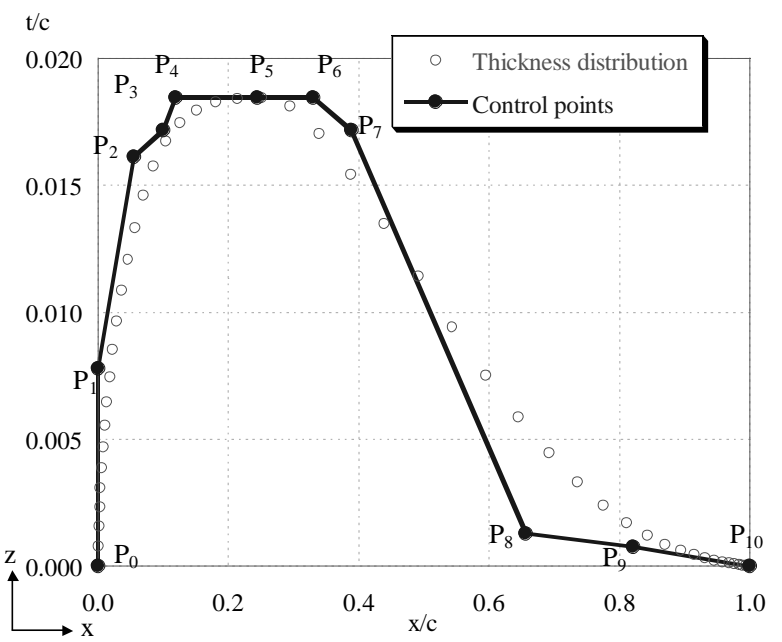

Fig. 17. Revised thickness definition
Table 3. Constraints for revised thickness definition

\begin{tabular}{l|c}
\hline Maximum thickness & $3<\mathrm{Z}_{\mathrm{P}_{5}}<4$ \\
\hline Maximum thickness location & $15<\mathrm{X}_{5}<70$ \\
\hline Continuous first derivative at $\mathrm{P}_{5}$ & $\mathrm{Z}_{\mathrm{P}_{4}}=\mathrm{Z}_{\mathrm{P}_{5}}=\mathrm{Z}_{\mathrm{P}_{6}}$ \\
\hline $\begin{array}{l}\text { Continuous second derivative } \\
\text { at } \mathrm{P}_{5}\end{array}$ & $\mathrm{XP}_{5}-\mathrm{X}_{\mathrm{P}_{3}}=\mathrm{X}_{7}-\mathrm{X}_{5}$, \\
\hline $\begin{array}{l}\text { Continuous first derivative } \\
\text { at leading edge }\end{array}$ & $\mathrm{Z}_{\mathrm{P}_{3}}=\mathrm{Z}_{7}$ \\
\hline
\end{tabular}

\subsection{Overview of Pareto Solutions}

The evolution was computed for 75 generations. After the computation, all the solutions evolved were sorted again to find the final Pareto solutions. The Pareto solutions were obtained in the four-dimensional objective function space. To understand the distribution of Pareto solutions, all Pareto solutions are projected into the two-dimensional objective function space between transonic and supersonic drag coefficients as shown in Fig. 18. In Fig. 18, Surface I shows the tradeoff between aerodynamic performances. The wings near Surface I have impractically large aspect ratios. The planform shapes of the extreme Pareto solutions that minimize the respective objective functions appear physically reasonable as shown in Fig. 19. They are more extreme than those in Fig. 10. A wing with the minimal transonic cruising drag has a less leading-edge sweep and a large aspect ratio. On the contrary, a wing with the lowest supersonic drag coefficient has a large leading-edge sweep to remain inside the Mach cone. The pitching moment is reduced by lowering the sweep angle and the wing chord length.

The results of the previous three-objective and the present four-objective optimization are compared here. The Pareto solutions obtained from both optimizations are plotted in Fig. 20. The present Pareto front is larger than three-objective's one, in particular, better tradeoff solutions appear in the tradeoff surface I compared to the previous tradeoff Surface I'. The region II, there are no solutions in the three-objective optimization. It might be due to the introduction of the pitching moment minimization. To examine it, all the present Pareto solutions in Fig. 18 are labeled by the bending and pitching moments, respectively, as shown in Fig. 21. The wings near the tradeoff surface between transonic and supersonic drag coefficients (tradeoff surface I in Fig. 18) have impractically large bending moments as shown in Fig. 21 (a). The bending moment is closely related to both transonic and supersonic drag coefficients. On the other hand, the pitching moment has an influence only on supersonic drag coefficient. As a consequence, the region II in Fig. 20 was primarily corresponding the minimization of the bending moment, not to the new objective function of the pitching moment minimization. The planform shapes that have the lowest bending moment obtained from 
the present and previous optimization are plotted in Fig. 22. Since these planform shapes are quite different, the present minimum wing and the wings belonged to the region II are found thanks to ARMOGAs. Similarly, the improvement of the present tradeoff surface I from the previous surface I' is due to ARMOGAs (Fig. 20).

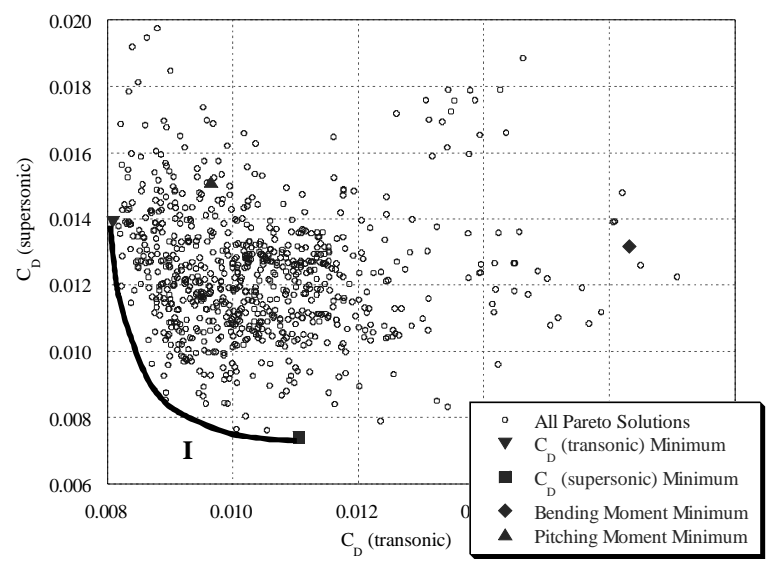

Fig. 18. Projection of Pareto solutions into two-dimensional plane between transonic and supersonic drag coefficients

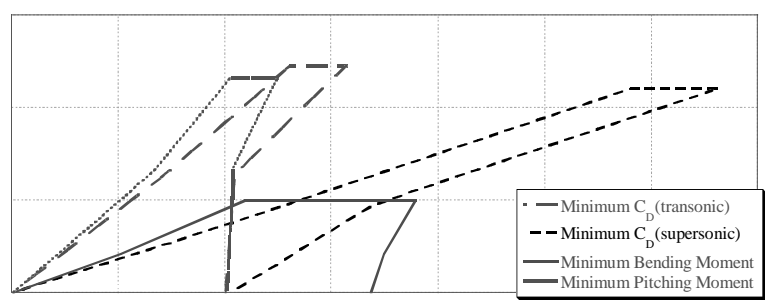

Fig. 19. Planform shapes of the extreme Pareto solutions

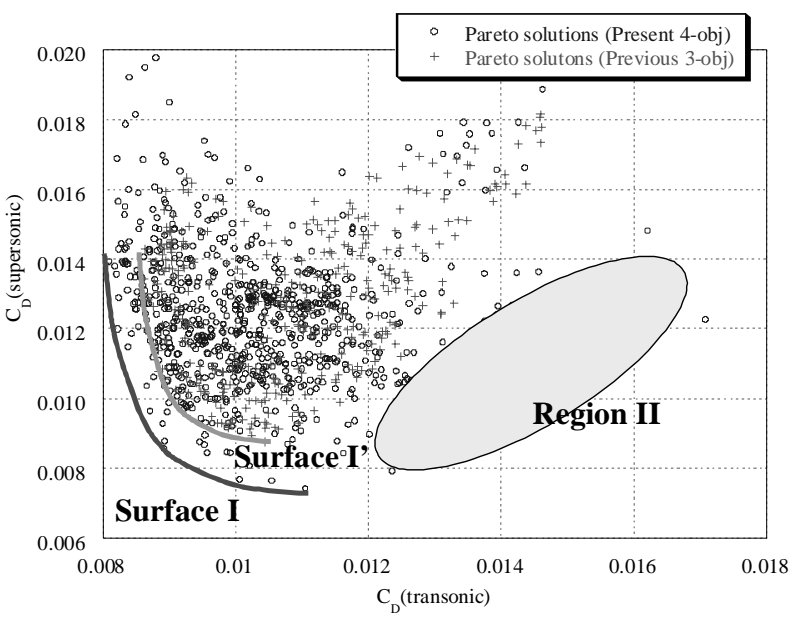

Fig. 20. Projection of Pareto solutions obtained by three-objective and four-objective optimizations into two-dimensional plane between transonic and supersonic drag coefficients

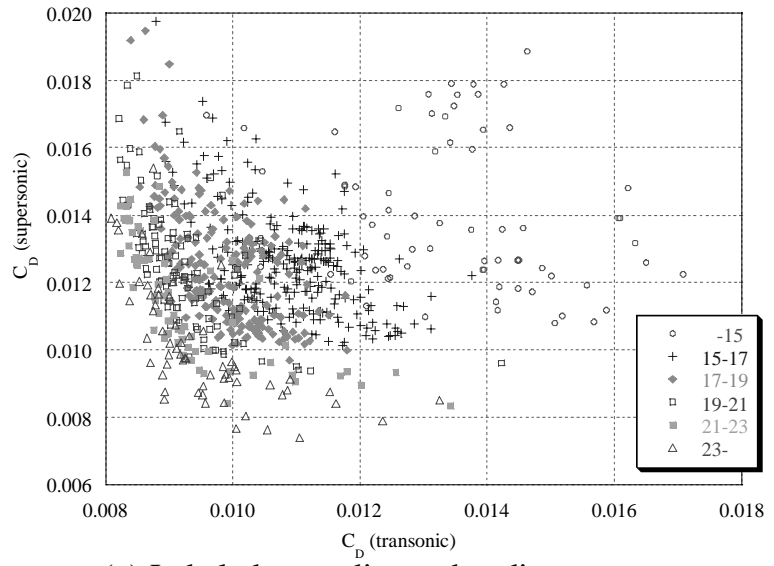

(a) Labeled according to bending moment

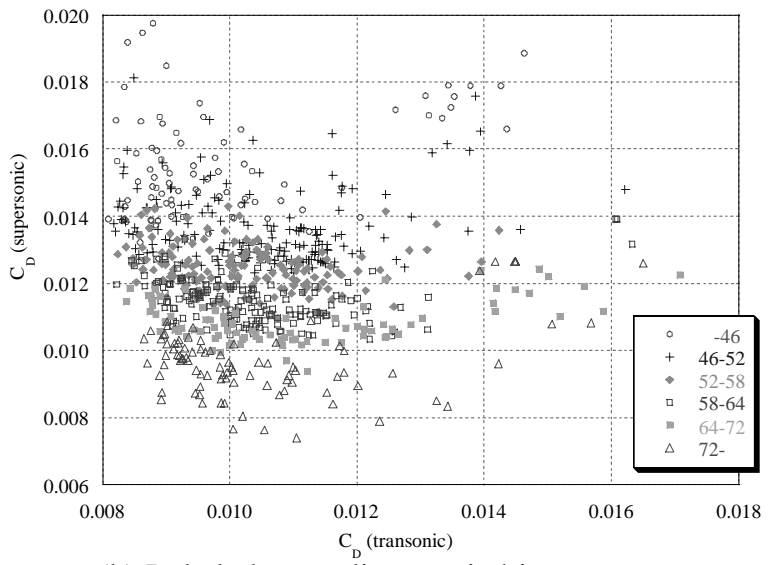

(b) Labeled according to pitching moment

Fig. 21. Projection of Pareto front to supersonic and transonic drag tradeoffs labeled according to bending and pitching moments.

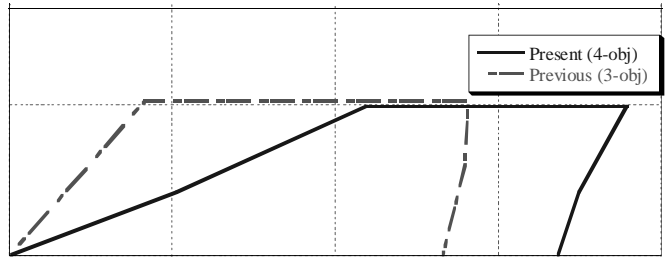

Fig. 22. Comparison of planform shapes having lowest bending moment obtained by the present and previous optimizations

\subsection{Comparison with NAL's Second Design and the Three-Objective Optimal Design}

To examine the quality of the present Pareto solutions, the selected wings are compared with NAL's second design as well as the previous wing obtained from the three-objective optimization. Table 4 summarizes aerodynamic performances of four wings compared: two present Pareto solutions (4V-A, $4 \mathrm{~V}-\mathrm{B})$, the previous Pareto solution (3V) and NAL's second design. All three Pareto solutions are superior to NAL's second design in all four objectives. Comparison of wing planform shapes is shown in Fig. 23. The present and previous planform shapes are similar to the "arrow wing" type. On the other hand, 
NAL's planform is similar to the conventional "delta wing" planform. These results indicate that the present arrow wing has good aerodynamic performance but doesn't have a large pitching moment because NAL's design has a higher pitching moment.

The thickness distributions at the wing root and $33 \%$ of the wingspan location of three Pareto solutions (4V-A, 4V-B, 3V) are presented in Fig. 24. In these figures, Pareto solutions $4 \mathrm{~V}-\mathrm{A}$ and $4 \mathrm{~V}-\mathrm{B}$ have much smoother thickness distributions than the previous Pareto solution of $3 \mathrm{~V}$. The present wings do not have a kink in the thickness distribution thanks to the improved parameterization, and less likely to cause a boundary layer separation. $\mathrm{C}_{\mathrm{p}}$ distributions and corresponding airfoil shapes at the wing root and $33 \%$ of the wingspan location are also shown in Fig. 25. Present $\mathrm{C}_{\mathrm{p}}$ distributions show much smoother distributions than the previous ones due to the smooth thickness distributions. From the present $C_{p}$ distributions, it is found that more lift is generated near the trailing edge because of the thicker thickness. Consequently, although the pitching moment minimization is introduced as the fourth objective function, pitching moments of present Pareto solutions are not dramatically improved.

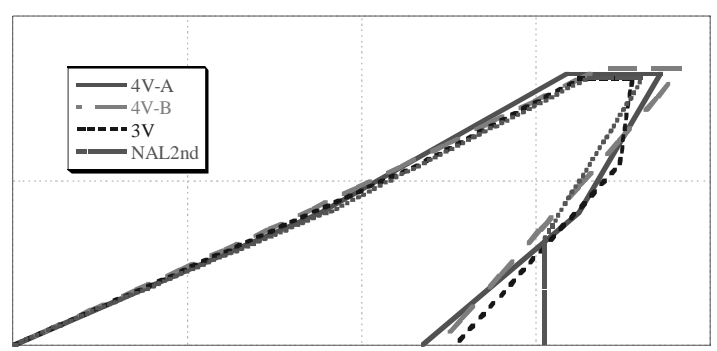

Fig. 23. Comparison of planform shapes among selected Pareto solutions and NAL's design

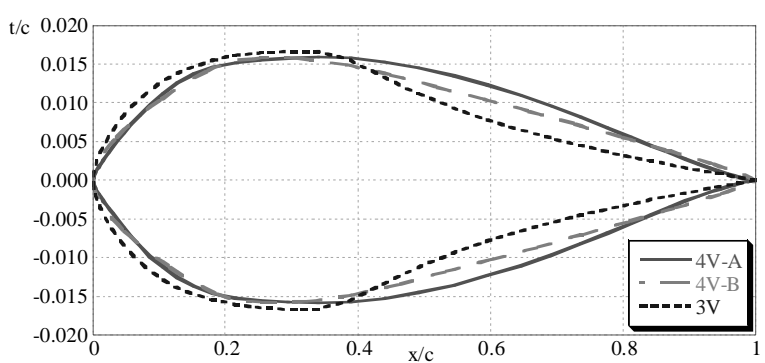

(a) Wing root

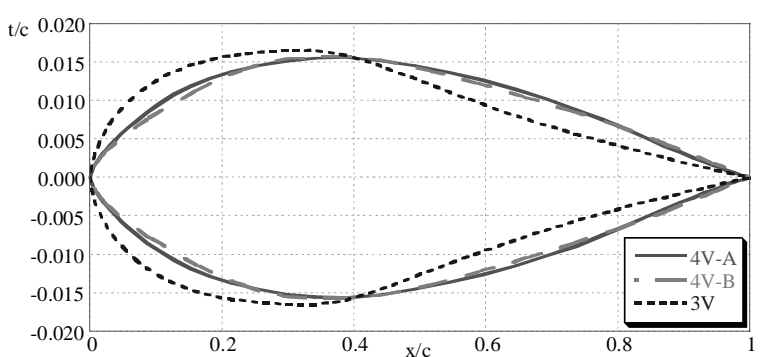

(b) $33 \%$ spanwise location

Fig. 24. Comparison of thickness distributions among selected Pareto solutions

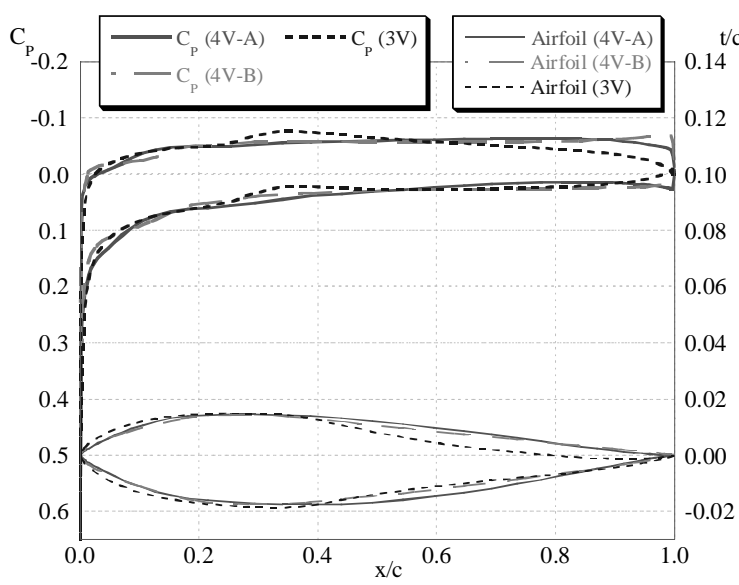

(a) Wing root

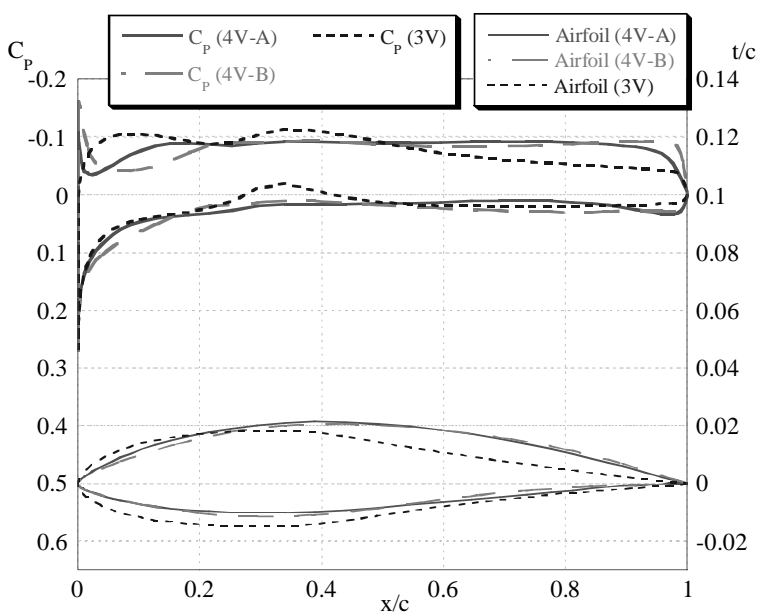

(b) $33 \%$ spanwise location

Fig. 25. Comparison of pressure distributions and airfoil shapes among selected Pareto solutions

Table 4. Aerodynamic performances

\begin{tabular}{c||c:c:c:c}
\hline & $\mathrm{C}_{\mathrm{D}, \mathrm{t}}$ & $\mathrm{C}_{\mathrm{D}, \mathrm{s}}$ & $\mathrm{M}_{\mathrm{B}}$ & $\mathrm{M}_{\mathrm{P}}$ \\
\hline 4V-A & 0.00998863 & 0.01085439 & 18.15 & 62.35 \\
4V-B & 0.01007195 & 0.01093646 & 17.39 & 60.60 \\
3V & 0.01004036 & 0.01093742 & 18.21 & 61.00 \\
NAL2nd & 0.01010175 & 0.01097646 & 18.23 & 63.31 \\
\hline
\end{tabular}

\section{Conclusion}

The multipoint design optimization of a wing for a SST has been performed by using MOGAs. Three objective functions were first used to minimize the supersonic drag, the transonic drag and the bending moment at the wing root. The complete wing shape was represented by in total of 66 design variables. The Navier-Stokes solver was used to evaluate the aerodynamic performances. Then, the revised optimization was performed to further improve the above optimization. The definition of the thickness distributions was improved and the minimization of the pitching moment was added to the objective functions. The resulting optimization contained four objective functions and 72 design variables. 
Successful optimization results are obtained in both cases. The planforms of the extreme Pareto solutions appear physically reasonable. Global tradeoffs between the objectives are presented.

In the case of the three-objective optimization, one Pareto solution has better performance in all three objective functions compared with NAL's second design. The comparison of the present Pareto solution with the optimal wing designed previously under the inviscid flow is also carried out to examine the viscous effect. The viscous effect is found to have a large influence on the thickness distribution. The present result is found better to prevent the possible boundary layer separation. The analysis of the Pareto solutions suggests that a desirable planform shape is the arrow wing with a relatively large taper ratio and a relatively small aspect ratio similar to the previous inviscid results.

Then the four-objective optimization was performed with smoother thickness definition by using ARMOGAs. The resulting Pareto front appeared better than the three-objective case thanks to the range adaptation. ARMOGAs are confirmed to work well in a large search space. By improving the definition of the thickness distributions, more realistic thickness distributions are obtained. The present Pareto solutions superior to NAL's second design in all four objective functions are examined in detail. As for the planform, optimal wings are similar to the "arrow wing" type. It also shows that the arrow wing maintains the pitching moment at reasonable levels especially below that of NAL's second design. The resulting arrow wing is considered as a good design candidate for the next-generation SST.

\section{Acknowledgements}

The present computation was carried out in parallel using ORIGIN2000 in the Institute of Fluid Science, Tohoku University. This research was partly funded by Japanese Government's Grants-in-Aid for Scientific Research, No. 10305071. The second author's research has been partly supported by Bombardier Aerospace, Toronto, Canada. The authors would like to thank National Aerospace Laboratory's SST Design Team for providing many useful data.

\section{References}

[1] Sakata, K., "Supersonic Experimental Airplane Program in NAL and its CFD-Design Research Demand," $2^{\text {nd }}$ SST-CFD Workshop, pp. 53-56, 2000.

[2] Vicini, A. and Quagliarella, D., "Multipoint Transonic Airfoil Design by Means of a Multiobjective Genetic Algorithm," AIAA 97-0082, 1997.
[3] Obayashi, S., Sasaki, D., Takeguchi, Y. and Hirose, N., "Multiobjective Evolutionary Computation for Supersonic Wing-Shape Optimization," IEEE Transactions on Evolutionary Computation, Vol. 4, No. 2, pp. 182-187, 2000.

[4] Sasaki, D., Obayashi, S., Sawada, K. and Himeno, R., "Multiobjective Aerodynamic Optimization of Supersonic Wings Using Navier-Stokes Equations," Proc. of ECCOMAS 2000 [CD-ROM], 2000.

[5] Fonseca, C. M. and Fleming, P. J., "Genetic Algorithms for Multiobjective Optimization: Formulation, Discussion and Generalization," Proc. of the $5^{\text {th }}$ ICGA, pp. 416-423, 1993.

[6] Obayashi, S., Takahashi, S. and Takeguchi, Y., "Niching and Elitist Models for MOGAs," Parallel Problem Solving from Nature - PPSN $\mathrm{V}$, Lecture Notes in Computer Science, Springer, Berlin Heidelberg New York, pp. 260-269, 1998.

[7] Eshelman, L. J. and Schaffer, J. D., "Real-coded genetic algorithms and interval schemata," Foundations of Genetic Algorithms2, Morgan Kaufmann Publishers, Inc., San Mateo, pp. 187-202, 1993.

[8] Arakawa, M. and Hagiwara, I., "Development of Adaptive Real Range (ARRange) Genetic Algorithms," JSME International Journal, Series C, Vol. 41, No. 4, pp. 969-977, 1998.

[9] Oyama, A., Obayashi, S. and Nakamura, S., "Real-Coded Adaptive Range Genetic Algorithm Applied to Transonic Wing Optimization," Lecture Notes in Computer Science, Vol. 1917, Springer-Verlag, Berlin Heidelberg New York, pp. 712-721, 2000.

[10] Obayashi, S. and Guruswamy, G. P., "Convergence Acceleration of an Aeroelastic Navier-Stokes Solver," AIAA Journal, Vol. 33 No. 6, pp. 1134-1141, 1995.

[11] Baldwin, B. S. and Lomax, H., "Thin Layer Approximation and Algebraic Model for Separated Turbulent Flows," AIAA 78-257, 1978.

[12] Jameson, A. and Caughey, D. A., "Effect of Artificial Diffusion Scheme on Multigrid Convergence," AIAA 77-635, 1977.

[13] Grenon, R., "Numerical Optimization in Aerodynamic Design with Application to a Supersonic Transport Aircraft," Proc. of International CFD Workshop for Super-Sonic Transport Design, pp. 83-104, 1997.

[14] Shimbo, Y., Yoshida, K., Iwamiya, T., Takaki, R. and Matsushima, K., "Aerodynamic Design of Scaled Supersonic Experimental Airplane," $I^{\text {st }}$ SST-CFD Workshop, pp. 62-67, 1998. 